Homology, Homotopy and Applications, vol.12(1), 2010, pp.93-110

\title{
ALGEBRAIC COBORDISM AND GROTHENDIECK GROUPS OVER SINGULAR SCHEMES
}

\author{
SHOUXIN DAI
}

\author{
(communicated by J. F. Jardine)
}

\begin{abstract}
A theorem of Levine-Morel states that algebraic cobordism groups are isomorphic to (multiplicative) Grothendieck groups over smooth schemes. We extend this theorem to singular schemes. As a consequence, we provide a new proof of the singular Riemann-Roch theorem of Baum-Fulton-MacPherson and a new type of Riemann-Roch theorem with respect to pullbacks of locally complete morphisms.
\end{abstract}

\section{Introduction}

Let $k$ be a field. Let us denote by $\mathrm{Sch}_{k}$ the category of separated $k$-schemes of finite type and by qSch ${ }_{k}$ (resp. $\mathrm{Sm}_{k}$ ) its full subcategory of quasi-projective (resp. smooth) $k$-schemes. By a smooth morphism in $\mathrm{Sch}_{k}$, we will always mean a smooth and quasiprojective morphism. In particular, a smooth $k$-scheme will always be assumed to be quasi-projective over $k$.

We recall that an oriented cohomology theory $A^{*}$ on $\mathrm{Sm}_{k}$ is a contravariant functor $X \mapsto A^{*}(X)$ sending $X \in \operatorname{Sm}_{k}$ to the category of graded commutative rings equipped with functorial push-forwards for projective morphisms, satisfying certain properties such as the projective bundle formula and homotopy. Please refer to [6, Def. 1.1.2] for full details.

An important feature of oriented cohomology theories is that they have a formal group law structure that describes how the first Chern classes behave with respect to the tensor product of line bundles. An oriented cohomology theory is called additive, multiplicative, and periodic if its formal group law is additive, multiplicative, and periodic respectively.

In [6], Levine and Morel construct a universal oriented cohomology theory on $\mathrm{Sm}_{k}$, called algebraic cobordism and written as $\Omega^{*}$, which is the algebro-geometric version of Quillen's complex cobordism. They show that $\Omega^{*}$ has the universal formal group law. That is to say, given a formal group law $\left(F_{R}, R\right)$, there is a unique homomorphism $\Omega^{*}(k) \rightarrow R$ sending $F_{\Omega}$ to $F_{R}$, which allows one to construct the universal theory with formal group law $\left(F_{R}, R\right)$ as $\Omega_{F}^{*}(X):=\Omega(X) \otimes_{\Omega(k)} R$. It is of particular interest when $R=\mathbb{Z}\left[\beta, \beta^{-1}\right]$. Let us use $\Omega_{\times}^{*}$ to denote $\Omega^{*} \otimes_{\Omega(k)} \mathbb{Z}\left[\beta, \beta^{-1}\right]$. It turns out that

Received May 5, 2009, revised Dec. 14, 2009; published on March 8, 2010.

2000 Mathematics Subject Classification: 14F99, 14C40.

Key words and phrases: algebraic cobordism, Grothendieck groups, singular schemes.

This article is available at http://intlpress.com/HHA/v12/n1/a8

Copyright (C) 2010, International Press. Permission to copy for private use granted. 
$\Omega_{\times}^{*}$ is the universal multiplicative periodic theory on $\mathrm{Sm}_{k}$. The homological notation for $\Omega_{\times}^{*}$ will be denoted by $\Omega_{*}^{\times}$.

Remark 1.1. For the construction of $\Omega_{*}$ (or equivalently, $\Omega^{*}$ ), please refer to $[\mathbf{6}, \S 2.4]$. However, for the purpose of this paper it suffices to know that $\Omega_{*}(X)$ (and $\Omega_{*}^{\times}(X)$ resp.) is essentially generated by $[f: Y \rightarrow X]$ (and $[f: Y \rightarrow X] \beta^{n}$ resp.), called cobordism cycles, with $Y$ being a smooth scheme and the morphism $f$ being projective.

We recall the following universal property of $K$-theory from [7]:

Theorem 1.2 (Levine-Morel). Let $A^{*}$ be a multiplicative periodic oriented cohomology theory on $\mathrm{Sm}_{k}$. Then there exists one, and only one, morphism of oriented cohomology theories $\operatorname{ch}_{A}: K_{0}\left[\beta, \beta^{-1}\right] \rightarrow A^{*}$, where $K_{0}\left[\beta, \beta^{-1}\right]=K_{0} \otimes_{\mathbb{Z}} \mathbb{Z}\left[\beta, \beta^{-1}\right]$.

By the universality of $\Omega_{\times}^{*}$ on $\mathrm{Sm}_{k}$, this yields:

Corollary 1.3 (Levine-Morel). Suppose that $k$ has characteristic zero. Then the canonical transformation $\Omega^{*} \rightarrow K_{0}\left[\beta, \beta^{-1}\right]$ descends to an isomorphism of multiplicative oriented cohomology theories $\Omega_{\times}^{*} \rightarrow K_{0}\left[\beta, \beta^{-1}\right]$ on $\mathrm{Sm}_{k}$.

It is natural to ask if this natural isomorphism over $\mathrm{Sm}_{k}$ can be extended to one over $\mathrm{Sch}_{k}$. For this purpose, it is necessary to replace oriented cohomology theories on $\mathrm{Sm}_{k}$ by oriented Borel-Moore homology theories on $\mathrm{Sch}_{k}$.

An oriented Borel-Moore homology theory $A_{*}$ on $\operatorname{Sch}_{k}$ is a functor $X \mapsto A_{*}(X)$ sending $X$ in $\operatorname{Sch}_{k}$ to the category of graded abelian groups with functorial pushforward for projective morphisms, and pullback maps for locally complete intersection (l.c.i.) morphisms, satisfying some natural axioms. See Definition 5.1.3 of [6] for details.

Note that on $\operatorname{Sch}_{k}, K$-theory shall be replaced by $G$-theory. Let us abbreviate the phrase Oriented Borel-Moore to OBM.

Remark 1.4. From Theorem 7.1.3 and Remark 4.1.12 of [6], $\Omega_{*}$ (and $\Omega_{*}^{\times}$resp.) is the universal OBM homology theory (and the universal multiplicative OBM homology theory resp.) on $\operatorname{Sch}_{k}$.

We are able to prove the following main result of this paper:

Theorem 1.5. Let $k$ be a field of characteristic zero. Then $G_{0}\left[\beta, \beta^{-1}\right]$ is the universal multiplicative OBM homology theory on $\mathrm{Sch}_{k}$. That is to say, for any multiplicative OBM homology theory $A_{*}$ on $\mathrm{Sch}_{k}$, there is a unique natural transformation of $\mathrm{OBM}$ homology theories $\tau: G_{0}\left[\beta, \beta^{-1}\right] \rightarrow A_{*}$.

In fact, the canonical natural transformation $\theta_{G}: \Omega_{*} \rightarrow G_{0}\left[\beta, \beta^{-1}\right]$ descends to a natural transformation of OBM homology theories on $\mathrm{Sch}_{k}$,

$$
\theta_{G}^{\times}: \Omega_{*}^{\times} \rightarrow G_{0}\left[\beta, \beta^{-1}\right],
$$

where for a scheme $X$ the map $\theta_{G}^{\times}$is defined by the following:

$$
[f: Y \rightarrow X] \beta^{n} \mapsto f_{*}\left[\mathcal{O}_{Y}\right] \beta^{n+\operatorname{dim}_{k} Y} .
$$


Remark 1.6. The transformation $\theta_{G}$ is natural by the universality of $\Omega_{*}$. As being natural only concerns commutativity with push-forwards but not the factor $\beta^{n}, \theta_{G}^{\times}$ is thus natural. On the other hand, the universality of $\Omega_{*}^{\times}$implies that $\theta_{G}^{\times}$is actually the unique natural transformation between the two theories, which is compatible with l.c. i. pullbacks and the first Chern class operators (i.e., a morphism of OBM homology theories).

We prove directly that the map (1) is an isomorphism on $\operatorname{Sch}_{k}$, which yields Theorem 1.5 via the universality of $\Omega_{*}^{\times}$.

We apply the main theorem to two situations. The first (Corollary 1.7) gives a new version of singular Riemann-Roch with respect to pullbacks by locally complete morphisms, and the second (Corollary 1.8) provides a new proof of the singular Riemann-Roch theorem of Baum-Fulton-MacPherson.

Corollary 1.7 (1.c. i. Riemann-Roch). Let $f: Y \rightarrow X \in \mathrm{qSch}_{k}$ be an l.c.i. morphsim of relative degree $d$. Then we have the following commutative diagram:

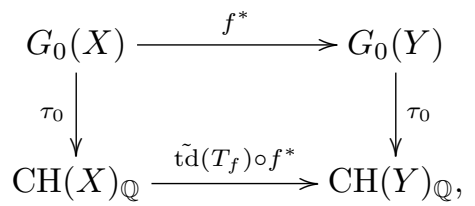

where, for a vector bundle $E \rightarrow Y$ over $Y, \tilde{\operatorname{td}}(E): \mathrm{CH}_{*}(Y)_{\mathbb{Q}} \rightarrow \mathrm{CH}_{*}(Y)_{\mathbb{Q}}$ sending $a \mapsto \operatorname{td}(E) \cap a$ by the cap-product map $\mathrm{CH}^{*}(Y)_{\mathbb{Q}} \otimes \mathrm{CH}_{*}(Y)_{\mathbb{Q}} \stackrel{\cap}{\rightarrow} \mathrm{CH}_{*}(Y)_{\mathbb{Q}}$ defined in [3].

Corollary 1.8 (Singular Riemann-Roch). Let $f: X \rightarrow Y$ be a projective morphism in $\mathrm{qSch}_{k}$. Then the following diagram is commutative:

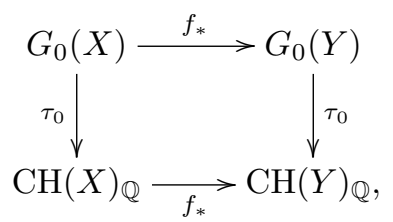

where $\tau_{0}$ is the restriction to degree zero of the natural transformation

$$
\tau: G_{0}\left[\beta, \beta^{-1}\right] \rightarrow \mathrm{CH} \otimes \mathbb{Q}\left[\beta, \beta^{-1}\right]^{(\mathrm{td})} .
$$

Moreover, $\tau_{0}$ coincides with the local Chern class morphism in [1].

\section{Acknowledgements}

Most of the work in this paper was done as part of my 2007 thesis. My sincere thanks go to M. Levine for his guidance and encouragement. I gratefully thank A. Merkurjev for his support. 


\section{Several lemmas}

This section provides several preliminary results needed for the proof of the main theorem.

Let us recall the following two localization theorems.

Theorem 2.1 (Quillen [8]). Let $X$ be a noetherian scheme, $i: Z \rightarrow X$ a closed immersion, and $j: U \rightarrow X$ the open complement of $Z$. Then there is a natural long exact sequence

$$
\begin{aligned}
\ldots \rightarrow G_{n}(Z) \stackrel{i_{*}}{\rightarrow} G_{n}(X) \stackrel{\stackrel{j^{*}}{\longrightarrow}}{\rightarrow} G_{n}(U) & \\
& \stackrel{\delta}{\rightarrow} G_{n-1}(Z) \rightarrow \ldots \rightarrow G_{1}(U) \\
& \stackrel{\delta}{\rightarrow} G_{0}(Z) \stackrel{i_{*}}{\rightarrow} G_{0}(X) \stackrel{j^{*}}{\longrightarrow} G_{0}(U) \rightarrow 0 .
\end{aligned}
$$

Theorem 2.2 (Levine-Morel [6]). Let $X$ be in $\operatorname{Sch}_{k}$. Let $i: Z \rightarrow X$ be a closed immersion and $j: U \rightarrow X$ the open complement. Then the sequence

$$
\Omega_{*}(Z) \stackrel{i_{*}}{\longrightarrow} \Omega_{*}(X) \stackrel{j^{*}}{\longrightarrow} \Omega_{*}(U) \rightarrow 0
$$

is exact.

As the tensor product is right exact, we have:

Corollary 2.3. Let $X$ be in $\operatorname{Sch}_{k}, i: Z \rightarrow X$ a closed immersion and $j: U \rightarrow X$ the open complement. Then the sequence $\Omega_{*}^{\times}(Z) \stackrel{i_{*}}{\longrightarrow} \Omega_{*}^{\times}(X) \stackrel{j^{*}}{\longrightarrow} \Omega_{*}^{\times}(U) \rightarrow 0$ is exact.

Throughout this section we assume that $k$ admits resolution of singularities, and we abbreviate $G_{0}(X)\left[\beta, \beta^{-1}\right]$ to $G_{0}(X)_{\beta}$.

Lemma 2.4. Take $X$ in $\operatorname{Sch}_{k}$. Let $i: X_{\text {red }} \rightarrow X$ be the reduction of $X$. Then the maps

$$
\begin{aligned}
i_{*}: \Omega_{*}^{\times}\left(X_{\mathrm{red}}\right) & \rightarrow \Omega_{*}^{\times}(X), \\
i_{*}: G_{0}\left(X_{\mathrm{red}}\right)_{\beta} & \rightarrow G_{0}(X)_{\beta}
\end{aligned}
$$

are isomorphisms.

Proof. The result for $G_{0}$ follows from Theorem 2.1 applied to $i: X_{\text {red }} \rightarrow X$, since the complement is empty.

For $\Omega_{*}^{\times}$, this follows from the same result for $\Omega_{*}$, which then follows directly from the definition.

Lemma 2.5. For $X \in \operatorname{Sch}_{k}$, the $\operatorname{map} \theta_{G}^{\times}(X): \Omega_{*}^{\times}(X) \rightarrow G_{0}(X)_{\beta}$ is surjective.

Proof. If $X$ is in $\operatorname{Sm}_{k}$, then we may use Theorem 1.2 and the fact that $K_{0}\left[\beta, \beta^{-1}\right]=$ $G_{0}\left[\beta, \beta^{-1}\right]$ on $\mathrm{Sm}_{k}$. 
In general, we may assume that $X$ is reduced. Then $X$ admits a filtration by reduced closed subschemes with $U_{l}:=X_{l} \backslash X_{l-1}$ in $\mathrm{Sm}_{k}$. In particular, $X_{0}$ is in $\operatorname{Sm}_{k}$ and the result is thus proven for $X_{0}$.

We have the commutative diagram

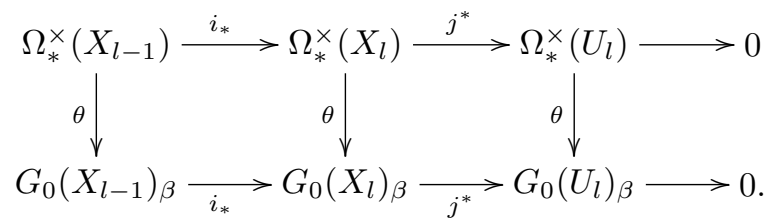

The rows are exact by Theorem 2.1 and Corollary 2.3. The result follows by induction on $l$ and a diagram chase.

Lemma 2.6. Let $p: V \rightarrow X$ be a vector bundle of rank $n+1$ in $\operatorname{Sch}_{k}$, and $q: P=$ $P(V) \rightarrow X$ the associated projective bundle. Then $q_{*}: \Omega_{*}(P) \rightarrow \Omega_{*}(X)$ is surjective.

Proof of the special case. Let us first prove the case where $V=X \times{ }_{k} \mathbb{A}^{n+1}$; thus $P=$ $X \times_{k} \mathbb{P}^{n}$. There is a closed immersion $i: X \rightarrow X \times_{k} \mathbb{P}^{n}$ such that $q \circ i=\mathrm{id}_{X}$. The composition of the induced morphisms

$$
q_{*} \circ i_{*}: \Omega_{*}(X) \rightarrow \Omega_{*}\left(X \times_{k} \mathbb{P}^{n}\right) \rightarrow \Omega_{*}(X)
$$

is the identity on $\Omega_{*}(X)$. It follows that $q_{*}$ is surjective. The lemma holds for this case.

Proof of the general case. Now let $V \rightarrow X$ be a general vector bundle of rank $n+1$. Let $Z$ be a proper closed subscheme of $X$ such that the restriction of $P$ to $U:=X \backslash Z$, the complement of $Z$ in $X$, is $U \times_{k} \mathbb{P}^{n}$. We denote by $P^{\prime}$ the restriction of $P$ to $Z$. We have the following commutative diagram of morphisms of localization sequences:

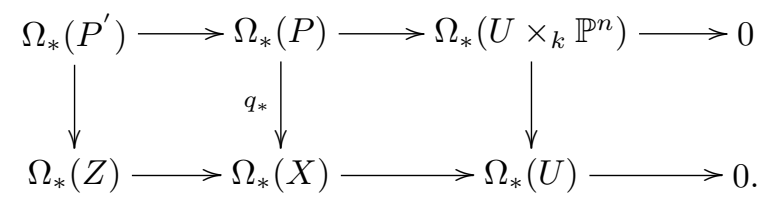

The vertical map on the left is surjective by induction on dimension of $X$, and the vertical map on the right is surjective as shown in the special case; we thus conclude that the map $q_{*}$ is surjective by the 5-lemma.

Lemma 2.7. Let $M$ be in $\mathrm{Sm}_{k}$ and $Z \subset M$ a reduced closed subscheme.

Consider the following commutative diagram:

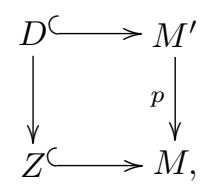

where $p$ is a sequence of blowups along smooth centers lying over $Z, D=p^{-1}(Z)$; 
then both vertical maps in the following commutative diagram are surjective:

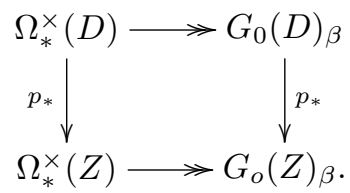

Proof. Since $p$ is a sequence of blowups along smooth centers lying over $Z$, it suffices to show that the lemma holds for the case where $M^{\prime}$ is the blowup of $M$ along some smooth subscheme $F$ of $Z$, as displayed in the following diagram:

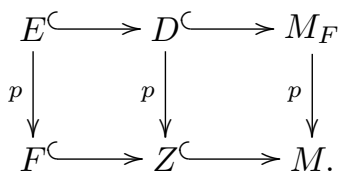

Let $U$ denote the complement of $F$ in $Z$, which is the same as the complement of $E$ in $D$. We then have the following commutative diagram, with the rows being the respective exact localization sequences:

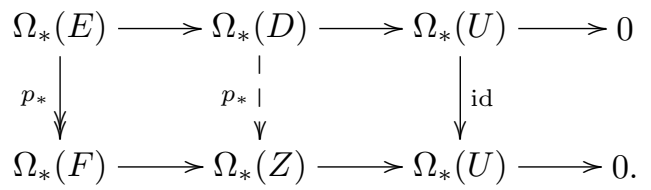

The map $p_{*}$ on the left is surjective by Lemma 2.6 as $p: E \rightarrow F$ is a projective bundle over $F$. The surjectivity of the dashed map $p_{*}$ then follows by the 5 -lemma.

The surjectivity of $G_{0}(D)_{\beta} \rightarrow G_{0}(Z)_{\beta}$ follows from the commutativity of the diagram.

Lemma 2.8. Let $D$ be a reduced finite type $k$-scheme, $D_{2}$ an irreducible component of $D$, and $D_{1}$ the union of the remaining irreducible components of $D$, so $D=D_{1} \cup D_{2}$. Let $D_{12}=D_{1} \cap D_{2}$ with inclusions $i_{j}: D_{12} \rightarrow D_{j}, \phi_{j}: D_{j} \rightarrow D$ for $j=1,2$. If we write $i_{*}^{-}=\left(i_{1 *},-i_{2 *}\right)$ and $\phi=\phi_{1 *}+\phi_{2 *}$, we have:

1. The sequence

$$
G_{0}\left(D_{12}\right)_{\beta} \stackrel{i_{*}^{-}}{\longrightarrow} G_{0}\left(D_{1}\right)_{\beta} \oplus G_{0}\left(D_{2}\right)_{\beta} \stackrel{\phi}{\longrightarrow} G_{0}(D)_{\beta} \rightarrow 0
$$

is exact.

2. The map $\phi: \Omega_{*}^{\times}\left(D_{1}\right) \oplus \Omega_{*}^{\times}\left(D_{2}\right) \rightarrow \Omega_{*}^{\times}(D)$ is surjective.

Proof of (1). Consider the morphism $p: D_{1} \amalg D_{2} \rightarrow D_{1} \cup D_{2}$ induced by closed embeddings $D_{j} \rightarrow D_{1} \cup D_{2}$ for $j=1,2$. Let $U_{j}:=D_{j} \backslash D_{12}$ with open immersions $\sigma_{j}: U_{j} \rightarrow D_{j}$ for $j=1,2$, and let $i: D_{12} \rightarrow D$ be the inclusion. We denote by $\sigma_{j}^{\prime}$ the open immersions $U_{j} \rightarrow D$ for $j=1,2$. Let $\sigma_{*}:=\sigma_{1 *} \oplus \sigma_{2 *}$ and $\sigma_{*}^{\prime}:=\left(\sigma_{1 *}^{\prime}, \sigma_{2 *}^{\prime}\right)$. 
Since

$$
D_{1} \amalg D_{2} \backslash D_{12} \amalg D_{12}=D \backslash D_{12}=U_{1} \amalg U_{2},
$$

we have the following morphism of localization sequences:

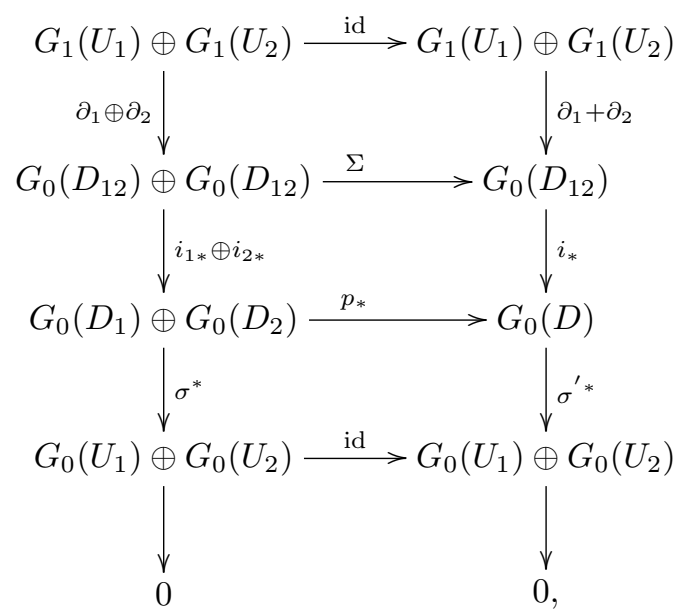

where $\Sigma$ is the sum map.

We note that

$$
\operatorname{ker}\left(p_{*}\right) \subset \operatorname{ker}\left(\sigma^{*} \circ p_{*}\right)=\operatorname{ker}\left(\sigma^{*}\right)=\operatorname{im}\left(i_{1 *} \oplus i_{2 *}\right) .
$$

Thus, if $y=y_{1} \oplus y_{2}$ is in $\operatorname{ker}\left(p_{*}\right)$, then there are elements $x_{i} \in G_{0}\left(D_{12}\right)$ with $y_{1}=i_{1 *}\left(x_{1}\right), y_{2}=i_{2 *}\left(x_{2}\right)$. Since $p_{*}\left(i_{1 *}\left(x_{1}\right) \oplus i_{2 *}\left(x_{2}\right)\right)=0$, we have $i_{*}\left(x_{1}+x_{2}\right)=0$; hence there are elements $\alpha_{i} \in G_{1}\left(U_{i}\right)$ with $\partial_{1}\left(\alpha_{1}\right)+\partial_{2}\left(\alpha_{2}\right)=x_{1}+x_{2}$. Replacing $x_{i}$ with $x_{i}-\partial_{i}\left(\alpha_{i}\right)$, we may assume that $x_{1}=-x_{2}$ in $G_{0}\left(D_{12}\right)$; i.e., there is an $x \in G_{0}\left(D_{12}\right)$ with

$$
y_{1}=i_{1 *}(x), y_{2}=-i_{2 *}(x)
$$

which proves the exactness of our sequence (1) at $G_{0}\left(D_{1}\right)_{\beta} \oplus G_{0}\left(D_{2}\right)_{\beta}$. The surjectivity of $\phi$ in (1) follows from diagram (2) and the 5-lemma, noting that the maps $\Sigma$ and id are surjective.

Proof of (2). Using the right exact localization sequence of $\Omega_{*}^{\times}$, the same argument as for the surjectivity in (1) applies to prove the surjectivity of $\phi$.

Lemma 2.9. Let $D$ be a strict normal crossing divisor on a scheme $M \in \mathrm{Sm}_{k}$. Then $\Omega_{*}^{\times}(D) \stackrel{\sim}{\rightarrow} G_{0}(D)_{\beta}$.

Proof. We may assume that $D$ is reduced.

Let us write $D=D_{1} \cup D_{2}$, where $D_{2}$ is an irreducible component of $D$. We proceed by induction on the number of irreducible components of $D$ as well as on the dimension of $D$. As in the previous Lemma 2.8, we write $D_{12}=D_{1} \cap D_{2}$, and use $i_{j}: D_{12} \rightarrow D_{j}$ and $\phi_{j}: D_{j} \rightarrow D$ for $j=1,2$ to denote the inclusions. 
We have the following commutative diagram:

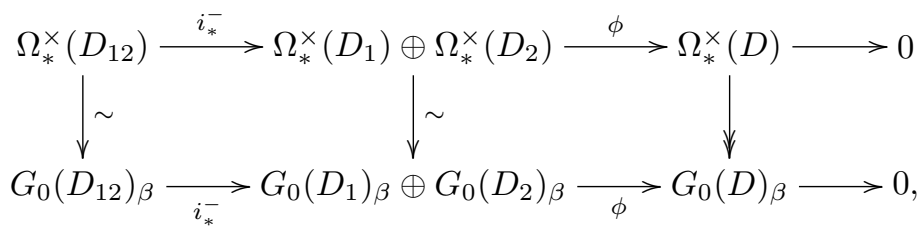

where $i_{*}^{-}=\left(i_{1 *},-i_{2 *}\right)$ and $\phi=\phi_{1 *}+\phi_{2 *}$. The first two of the three vertical maps are isomorphisms by induction, while the third one is surjective. Clearly the top row is a complex; in addition, the bottom row is exact by Lemma 2.8(1) and the top map $\phi$ is surjective by Lemma $2.8(2)$.

We fill $K:=\operatorname{coker}\left(i_{*}\right)$ into the following diagram:

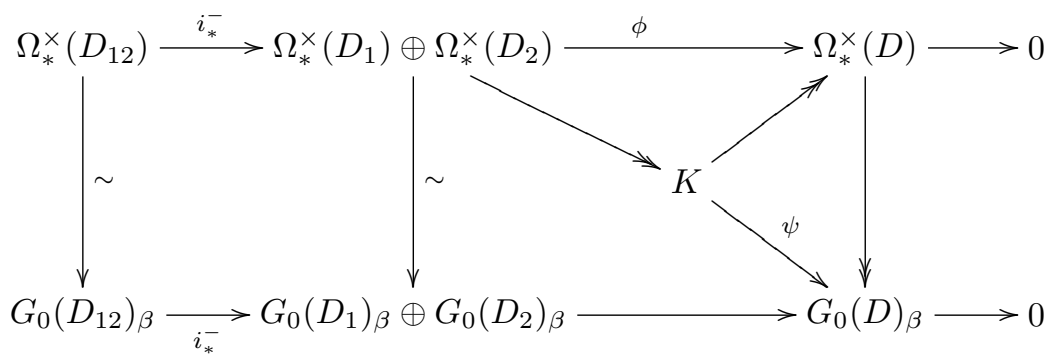

with the sequence

$$
\Omega_{*}^{\times}\left(D_{12}\right) \rightarrow \Omega_{*}^{\times}\left(D_{1}\right) \oplus \Omega_{*}^{\times}\left(D_{2}\right) \rightarrow K \rightarrow 0
$$

being exact. Since $\phi \circ i_{*}=0$, we have a surjective map $K \rightarrow \Omega_{*}^{\times}(D)$. By the 5 -lemma $\psi: K \rightarrow G_{0}(D)_{\beta}$ is an isomorphism; hence the surjection $\Omega_{*}^{\times}(D) \rightarrow G_{0}(D)_{\beta}$ is an isomorphism.

Lemma 2.10. Let $M$ be in $\mathrm{Sm}_{k}$ and let $Z \subset M$ a reduced closed subscheme. Let $F \subset M$ be a smooth closed subscheme contained in $Z$. We denote by $M_{F}$ the blowup of $M$ along $F$ with the canonical projective morphism $p: M_{F} \rightarrow M$. Then the sequence

$$
0 \rightarrow \operatorname{ker}\left(p_{*}\right) \rightarrow G_{n}\left(M_{F}\right) \stackrel{p_{*}}{\rightarrow} G_{n}(M) \rightarrow 0
$$

is split exact.

Proof. It suffices to show that $p_{*} \circ p^{*}=$ id on $G_{n}(M)=K_{n}(M)$. We have the projection formula

$$
p_{*}\left(a \cdot p^{*}(b)\right)=p_{*}(a) \cdot b
$$

for all $a \in K_{0}\left(M_{F}\right)$ and $b \in G_{n}(M)$. Thus, for any $x \in G_{n}(M)$, we have

$$
p_{*}\left(p^{*}(x)\right)=p_{*}\left(\left[\mathcal{O}_{M_{F}}\right] \cdot p^{*}(x)\right)=p_{*}\left(\left[\mathcal{O}_{M_{F}}\right]\right) \cdot x .
$$

However, $R^{q} p_{*}\left(\mathcal{O}_{M_{F}}\right)=0$ for $q>0$, and $p_{*}\left(\mathcal{O}_{M_{F}}\right)=\mathcal{O}_{M}$; so $p_{*}\left(\left[\mathcal{O}_{M_{F}}\right]\right)=\left[\mathcal{O}_{M}\right]$, and $p_{*}\left(p^{*}(x)\right)=\left[\mathcal{O}_{M}\right] \cdot x=x$. 
Lemma 2.11. Borrowing notation from the preceding Lemma 2.10, we denote by D the exceptional divisor $p^{-1}(F)$.

Let $K$ be the kernel of

$$
p_{*}: G_{0}\left(M_{F}\right) \rightarrow G_{0}(M)
$$

and $K^{\prime}$ be the kernel of

$$
p_{*}: G_{0}(D) \rightarrow G_{0}(Z) .
$$

Then the inclusion $i: D \rightarrow M_{F}$ induces an isomorphism $K^{\prime} \simeq K$.

Proof. Let us look at the following diagram:

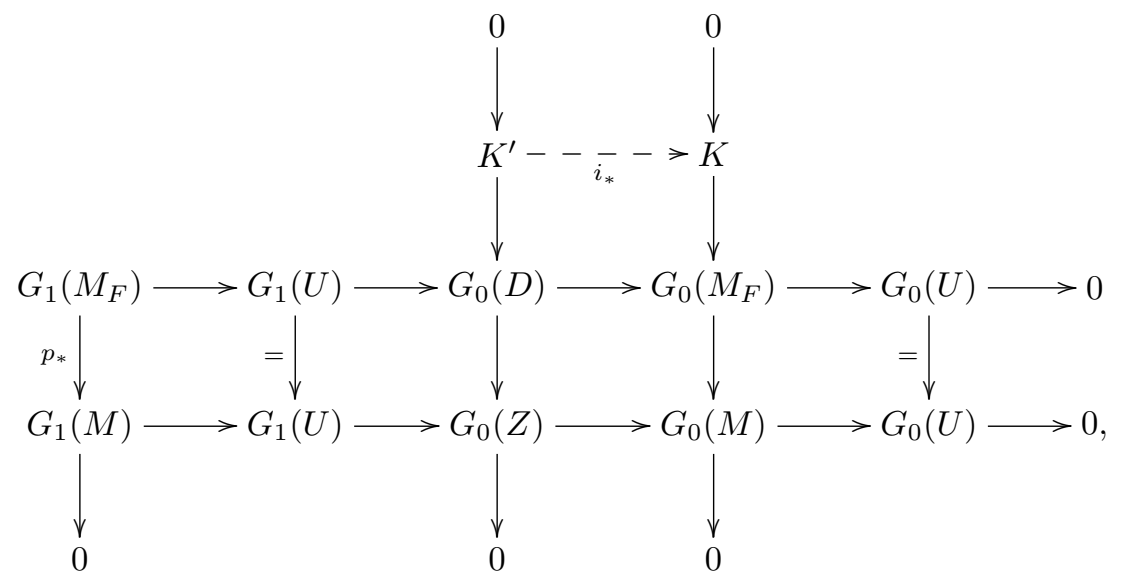

where $i_{*}$ is the natural map induced by $i: D \rightarrow M_{F}$ and the rows are the respective localization sequences.

Surjectivity of $i_{*}$ : To see this, we pick an element $a$ in $K$. It goes to 0 in $G_{0}(M)$ and thus goes to 0 in $G_{0}(U)$ as well by the commutativity of the diagram. Exactness implies that there is an element of $b$ of $G_{0}(D)$ whose image in $G_{0}\left(M_{F}\right)$ is $a$. Let $c$ be the image of $b$ in $G_{0}(Z)$. Then $c$ goes to 0 in $G_{0}(M)$, so it comes from an element $d$ in $G_{1}(U)$. Let $e$ be the image of $d$ in $G_{0}(D)$. Then $b-e$ belongs to $K^{\prime}$ and its image in $K$ is $a$.

Injectivity of $i_{*}$ : Let $x$ be such an element that $i_{*}(x)=0$. Then it is the image of some element $y$ in $G_{1}(U)$, which goes to 0 in $G_{0}(Z)$ by commutativity. Therefore, $y$ is the image of some element $z$ in $G_{1}(M)$. Since $p_{*}$ is split surjective by Lemma 2.10, we can lift $z$ to an element $\tilde{z}$ in $G_{1}\left(M_{F}\right)$, whose image in $G_{1}(U)$ is $y$. Therefore, $x$ is the image of $\tilde{z}$ in $G_{0}(D)$, which is then 0 . We conclude that $i_{*}$ is injective. 
Remark 2.12. Let us consider the following localization commutative diagrams:
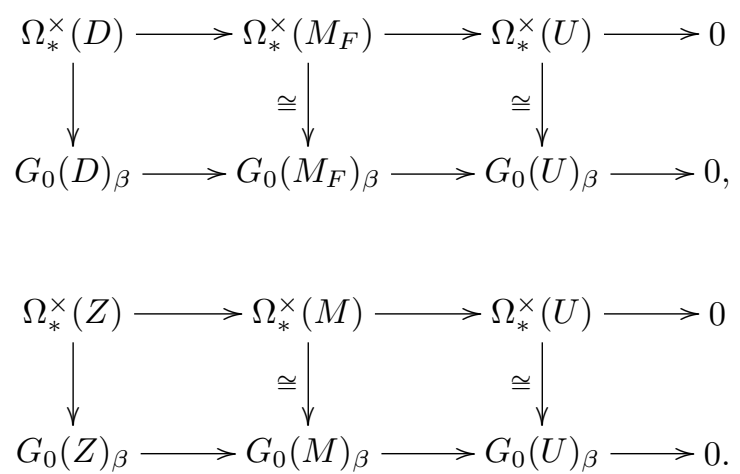

From Lemma 2.11 it is easy to deduce that

$$
\operatorname{ker}\left(\Omega_{*}^{\times}(D) \rightarrow \Omega_{*}^{\times}(Z)\right) \rightarrow \operatorname{ker}\left(\Omega_{*}^{\times}\left(M_{F}\right) \rightarrow \Omega_{*}^{\times}(M)\right)
$$

is surjective. This is because Lemma 2.11 still holds if we replace $Z$ by $F$, and $D$ by $E:=p^{-1}(F)$; i.e., the map

$$
\operatorname{ker}\left(G_{0}(E)_{\beta} \rightarrow G_{0}(F)_{\beta}\right) \rightarrow \operatorname{ker}\left(G_{0}\left(M_{F}\right)_{\beta} \rightarrow G_{0}(M)_{\beta}\right)
$$

is an isomorphism. We can replace the $G_{0}\left[\beta, \beta^{-1}\right]$ by $K_{0}\left[\beta, \beta^{-1}\right]$ since everything is smooth; similarly, $K_{0}\left[\beta, \beta^{-1}\right]$ is isomorphic to theory $\Omega_{*}^{\times}$by Corollary 1.3. Thus

$$
\operatorname{ker}\left(\Omega_{*}^{\times}(E) \rightarrow \Omega_{*}^{\times}(F)\right) \rightarrow \operatorname{ker}\left(\Omega_{*}^{\times}\left(M_{F}\right) \rightarrow \Omega_{*}^{\times}(M)\right)
$$

is an isomorphism. Since the map

$$
\operatorname{ker}\left(\Omega_{*}^{\times}(E) \rightarrow \Omega_{*}^{\times}(F)\right) \rightarrow \operatorname{ker}\left(\Omega_{*}^{\times}\left(M_{F}\right) \rightarrow \Omega_{*}^{\times}(M)\right)
$$

factors through

$$
\operatorname{ker}\left(\Omega_{*}^{\times}(D) \rightarrow \Omega_{*}^{\times}(Z)\right),
$$

the surjectivity of

$$
\operatorname{ker}\left(\Omega_{*}^{\times}(D) \rightarrow \Omega_{*}^{\times}(Z)\right) \rightarrow \operatorname{ker}\left(\Omega_{*}^{\times}\left(M_{F}\right) \rightarrow \Omega_{*}^{\times}(M)\right)
$$

follows.

\section{Main theorem}

Let $Z$ be a $k$-scheme which admits an embedding into some smooth $k$-scheme $M$. By Hironaka [4], there is a sequence of blowups of $M, p: M^{\prime} \rightarrow M$, along smooth centers lying over $Z$ such that $D:=p^{-1}(Z)$ is a strict normal crossing divisor of $M^{\prime}$.

To be more precise, we have the following diagram of blowups:

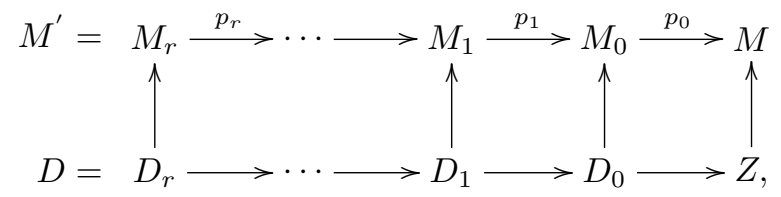


where

- $p_{i+1}: M_{i+1} \rightarrow M_{i}$ is the blowup of $M_{i}$ along some smooth $F_{i} \subset D_{i}$ for $i=0, \ldots, r-1$,

- $D_{i+1}=p_{i+1}^{-1}\left(D_{i}\right)$ for $i=0, \ldots, r-1$,

- $p=p_{0} \circ \ldots \circ p_{r}$.

Lemma 3.1. In the commutative diagram of short exact sequences,

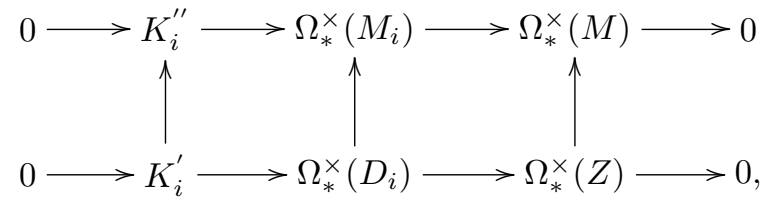

the $\operatorname{map} K_{i}^{\prime} \rightarrow K_{i}^{\prime \prime}$ is surjective for all $i=0, \ldots, r$.

In particular, $K_{r}^{\prime} \rightarrow K_{r}^{\prime \prime}$ is surjective.

Proof. We proceed by induction.

For $i=0, p_{0}$ is only a single blowup, and the claim follows from Remark 2.12. Let us assume the claim for $i \geqslant 0$. We must show that the claim holds for $i+1$.

Note that $K_{i+1}^{\prime} \rightarrow K_{i}^{\prime}$ is surjective by Lemma 2.7 applied to $p_{i+1}$, and $K_{i+1}^{\prime \prime} \rightarrow K_{i}^{\prime \prime}$ is surjective since $p_{i+1 *}: G_{0}\left(M_{i+1}\right) \rightarrow G_{0}\left(M_{i}\right)$ is (split) surjective and $G_{0}\left(M_{j}\right)_{\beta}=$ $\Omega_{*}^{\times}\left(M_{j}\right)$ as $M_{j}$ is smooth. Letting

$$
N^{\prime}:=\operatorname{ker}\left(\Omega_{*}^{\times}\left(D_{i+1}\right) \rightarrow \Omega_{*}^{\times}\left(D_{i}\right)\right) \quad \text { and } \quad M^{\prime}:=\operatorname{ker}\left(\Omega_{*}^{\times}\left(M_{i+1}\right) \rightarrow \Omega_{*}^{\times}\left(M_{i}\right)\right),
$$

then we have the natural morphism of short exact sequences as follows:

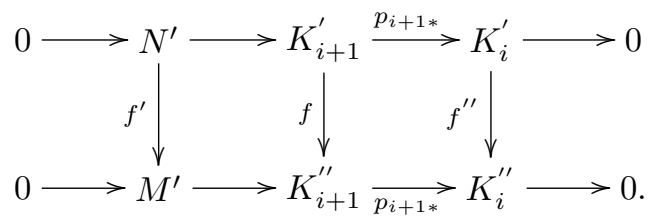

We see that $f^{\prime}$ is surjective because $p_{i+1}$ is a single blowup, and that $f^{\prime \prime}$ is surjective by induction. The lemma thus follows by the 5-lemma.

Lemma 3.2. In the commutative diagram of short exact sequences,

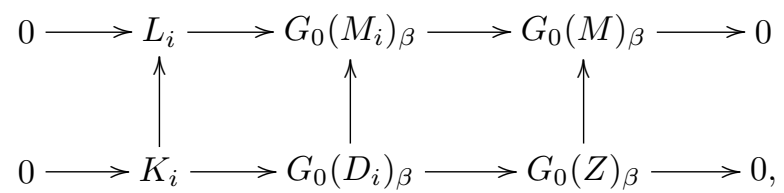

the $\operatorname{map} K_{i} \rightarrow L_{i}$ is an isomorphism for all $i=0, \ldots, r$.

In particular $K_{r} \rightarrow L_{r}$ is an isomorphism.

Proof. The same argument applies as in the preceding lemma using the isomorphism of Lemma 2.11 instead of the surjection of Remark 2.12. 
Theorem 3.3. $\theta_{G}^{\times}(Z): \Omega_{*}^{\times}(Z) \rightarrow G_{0}(Z)_{\beta}$ is an isomorphism.

Proof. We have the following commutative diagram:

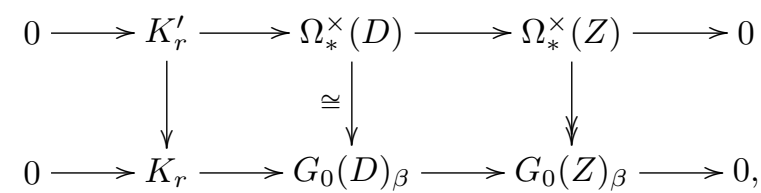

where the middle map is an isomorphism by Lemma 2.9. It follows that $K_{r}^{\prime} \rightarrow K_{r}$ is injective.

By Claims 3.1 and 3.2, we have the isomorphism $K_{r} \simeq L_{r}$ and the epimorphism $K_{r}^{\prime} \rightarrow K_{r}^{\prime \prime}$. Moreover, $K_{r}^{\prime \prime} \simeq L_{r}$ because $M$ and $M^{\prime}$ are both smooth.

We conclude that $K_{r}^{\prime} \rightarrow K_{r}$ is surjective in view of the following commutative diagram:

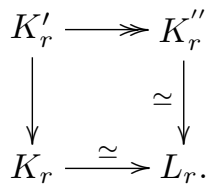

Therefore, $K_{r}^{\prime} \simeq K_{r}$ which implies that $\Omega_{*}^{\times}(Z) \simeq G_{0}(Z)_{\beta}$. This completes the proof that the natural transformation (1) is an isomorphism. As we have already remarked, this proves Theorem 1.5.

Remark 3.4. From the proof of the theorem, it is easy to see that the isomorphism $\Omega_{*}^{\times}(Z) \simeq G_{0}(Z)_{\beta}$ does not depend on the choice of embeddings $Z \hookrightarrow M$, nor does it depend on the choice of the resolution blowup sequences. This is because what we have proved is actually only the injectivity of the canonical surjective map $\Omega_{*}^{\times}(Z) \rightarrow G_{0}(Z)_{\beta}$.

\section{Applications: Riemann-Roch}

4.1. l.c.i. R.R.

Let $A_{*}$ be an OBM homology theory. We recall briefly how to twist $A_{*}$ into a new OBM theory. Please refer to $\S 8.2$ of [5] and $\S 10.5$ of [7] for details.

Let $\tau=\left(\tau_{i}\right) \in \prod_{i=0}^{\infty} A_{i}(k)$, with $\tau_{0}=1$. Following Levine and Morel, one can twist $A_{*}$ by $\tau$ as follows:

The groups and push-forward maps are unchanged:

$$
A_{*}^{(\tau)}(X):=A_{*}(X), f_{*}^{(\tau)}=f_{*} .
$$

To define the twisting of the pullback for an l.c.i. morphism $f: X \rightarrow Y$, let us choose a factorization of $f$ as $f=q i$, with $i: Y \rightarrow P$ a regular embedding and $q: P \rightarrow X$ a smooth morphism. We have the relative tangent bundle $T_{q} \rightarrow P$, defined as the vector bundle whose dual has sheaf of sections the relative differentials $\Omega_{Y / X}^{1}$. Letting $\mathcal{I}$ be the ideal sheaf of $Y$ in $P$, we let $N_{i} \rightarrow Y$ be the bundle whose dual has sheaf of sections $\mathcal{I} / \mathcal{I}^{2}$. We let $\left[N_{f}\right] \in K^{0}(Y)$ be the class $\left[N_{i}\right]-\left[i^{*} T_{q}\right]$. We call $\left[N_{f}\right]$ 
the virtual normal bundle of $f: Y \rightarrow X$. It is easy to see that $\left[N_{f}\right]$ is independent of the choice of the factorization of $f$.

We define

$$
f_{(\tau)}^{*}:=\tilde{c}_{\tau}\left(N_{f}\right) \circ f^{*}
$$

and for any line bundle $L$ over $X$, we set

$$
\tilde{c}_{1}^{(\tau)}(L):=\tilde{c}_{\tau}(L) \circ \tilde{c}_{1}(L)
$$

where for a vector bundle $E \rightarrow X$, the construction $\tilde{c}_{\tau}(E)$ is given by Lemma 8.1 of $[5]$.

Remark 4.1. This does define a new oriented Borel-Moore homology theory on $\operatorname{Sch}_{k}$, denoted by $A_{*}^{(\tau)}$. The definition of $\tilde{c}_{1}^{(\tau)}(L)$ can be rewritten as $\tilde{c}_{1}^{(\tau)}(L)=\lambda_{(\tau)}\left(\tilde{c}_{1}(L)\right)$, where $\lambda_{(\tau)}(u)=\sum_{i \geqslant 0} \tau_{i} \cdot u^{i+1} \in A_{*}(k)[[u]]$. It is then clear that to give a twisting is equivalent to giving a formal series $\lambda_{(\tau)}(u)$ with leading term $u$.

Example 4.2. The Chow theory $\mathrm{CH}_{*}$ has the structure of OBM homology theory on $\mathrm{Sch}_{k}$. We give $\mathrm{CH}_{*} \otimes \mathbb{Q}\left[\beta, \beta^{-1}\right]$ the structure of OBM homology theory on $\mathrm{Sch}_{k}$ by taking the $\mathbb{Q}\left[\beta, \beta^{-1}\right]$-linear extension; i.e.,

$$
f_{\mathrm{CH}_{*} \otimes \mathbb{Q}\left[\beta, \beta^{-1}\right]}^{*}:=f_{\mathrm{CH}}^{*} \otimes \mathrm{id}
$$

and similarly for all other structures.

We can produce a new theory on $\mathrm{Sch}_{k}$, denoted by $\mathrm{CH}_{*} \otimes \mathbb{Q}\left[\beta, \beta^{-1}\right]^{(\mathrm{td})}$, by applying our twisting for the family $\tau$ given by

$$
\tau=\lambda_{(\tau)}(u)=\left(1-e^{-\beta u}\right) / \beta .
$$

In effect, the presence of the exponential term $e^{-\beta u}$ converts the additive OBM homology theory $\mathrm{CH}_{*} \otimes \mathbb{Q}\left[\beta, \beta^{-1}\right]$ into a multiplicative one $\mathrm{CH}_{*} \otimes \mathbb{Q}\left[\beta, \beta^{-1}\right]^{(\mathrm{td})}$ on $\mathrm{Sch}_{k}$ with the multiplicative formal group law

$$
F_{\mathrm{CH}}^{(\mathrm{td})}=u+v-\beta u v .
$$

Corollary 4.3. Suppose that $k$ admits resolution of singularities. Then there is a unique natural transformation of OBM homology theories on $\mathrm{Sch}_{k}$

$$
\tau: G_{0}\left[\beta, \beta^{-1}\right] \rightarrow \mathrm{CH}_{*} \otimes \mathbb{Q}\left[\beta, \beta^{-1}\right]^{(\mathrm{td})} .
$$

Proof. By Theorem $1.5 G_{0}\left[\beta, \beta^{-1}\right]$ is the universal periodic multiplicative OBM homology theory on $\mathrm{Sch}_{k}$. Thus, for any oriented OBM homology theory $A_{*}$ on $\mathrm{Sch}_{k}$ with periodic multiplicative formal group law, there exists a unique natural transformation $\tau: G_{0}\left[\beta, \beta^{-1}\right] \rightarrow A_{*}$.

By construction, $\mathrm{CH}_{*} \otimes \mathbb{Q}\left[\beta, \beta^{-1}\right]^{(\mathrm{td})}$ is an OBM theory on $\mathrm{Sch}_{k}$ with multiplicative periodic formal group law.

Thus we have a unique natural transformation of OBM homology theories

$$
\tau: G_{0}\left[\beta, \beta^{-1}\right] \rightarrow \mathrm{CH}_{*} \otimes \mathbb{Q}\left[\beta, \beta^{-1}\right]^{(\mathrm{td})} .
$$

Remark 4.4. For a vector bundle $E$ on $X$, we have the degree 0 endomorphism $\tilde{c}_{(\mathrm{td})^{-1}}(E)$ on $\mathrm{CH}_{*}(X)\left[\beta, \beta^{-1}\right]$. We can identify $\mathrm{CH}_{*}(X)$ with the degree 0 part 
of $\mathrm{CH}_{*}(X)\left[\beta, \beta^{-1}\right]$ by sending $x \in \mathrm{CH}_{p}(X)$ to $x \beta^{-p}$. We denote the restriction of $\tilde{c}_{(\mathrm{td})^{-1}}(E)$ to $\mathrm{CH}_{*}(X)$ by $\tilde{\mathrm{td}} E$. It follows that $\tilde{\mathrm{td}}(E)$ agrees with the classical Todd class automorphism of $\mathrm{CH}_{*}(X)$ as defined in [2].

Corollary 4.5 (1.c. i. Riemann-Roch). Let $f: Y \rightarrow X \in \mathrm{qSch}_{k}$ be an l.c.i. morphsim of relative degree $d$. Then we have the following commutative diagram:

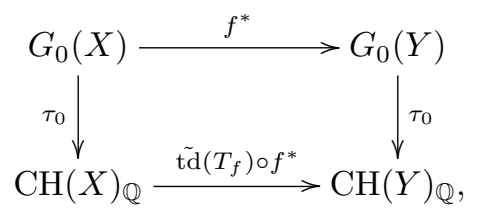

where, for a vector bundle $E \rightarrow Y$ over $Y$, tdd $(E): \mathrm{CH}_{*}(Y)_{\mathbb{Q}} \rightarrow \mathrm{CH}_{*}(Y)_{\mathbb{Q}}$ sending $a \mapsto \operatorname{td}(E) \cap$ a by cap-product map $\mathrm{CH}^{*}(Y)_{\mathbb{Q}} \otimes \mathrm{CH}_{*}(Y)_{\mathbb{Q}} \stackrel{\cap}{\rightarrow} \mathrm{CH}_{*}(Y)_{\mathbb{Q}}$ defined in [3].

Proof. By Corollary 4.3, there is a natural transformation of OBM homology theories

$$
\tau: G_{0}\left[\beta, \beta^{-1}\right] \rightarrow \mathrm{CH}_{*} \otimes \mathbb{Q}\left[\beta, \beta^{-1}\right]^{(\mathrm{td})} .
$$

By restricting $\tau$ to degree zero, denoted by $\tau_{0}$, the naturality of $\tau$ gives us the following commutative diagram for an l.c.i. morphism $f: Y \rightarrow X \in \mathrm{qSch}_{k}$ :

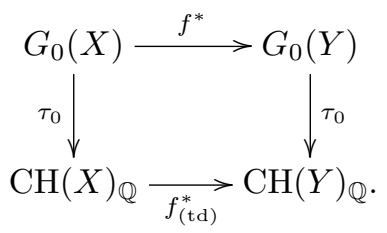

To finish the proof, it remains to verify that

$$
f_{(\mathrm{td})}^{*}=\tilde{\mathrm{td}}\left(T_{f}\right) \circ f^{*} .
$$

By definition,

$$
f_{(\mathrm{td})}^{*}:=\tilde{c}_{\mathrm{td}}\left(N_{f}\right) \circ f^{*} .
$$

Since $N_{f}=-T_{f}$ in $K_{0}(Y)$, and since $\tilde{\operatorname{td}}\left(T_{f}\right)$ is the restriction of $\tilde{c}_{(\mathrm{td})^{-1}}\left(T_{f}\right)$ to the degree zero portion, it suffices to show that

$$
\tilde{c}_{(\mathrm{td})}\left(-T_{f}\right)=\tilde{c}_{(\mathrm{td})^{-1}}\left(T_{f}\right) .
$$

But by definition of $(\tau)^{-1}$ and the multiplicative properties of $\tilde{c}_{\tau}$, we have

$$
\tilde{c}_{(\tau)^{-1}}(E)=\tilde{c}_{\tau}(E)^{-1}
$$

for all $\tau$ and $E$. Since $\tilde{c}_{\tau}(E)$ is multiplicative in $E$, we thus have

$$
\tilde{c}_{(\tau)^{-1}}(E)=\tilde{c}_{\tau}(E)^{-1}=\tilde{c}_{\tau}(-E) .
$$


ALGEBRAIC COBORDISM AND GROTHENDIECK GROUPS OVER SINGULAR SCHEMES 107

\subsection{Singular R.R.}

Corollary 4.6 (Singular R.R.). Let $f: X \rightarrow Y$ be a projective morphism in $\mathrm{qSch}_{k}$. Then the following diagram is commutative:

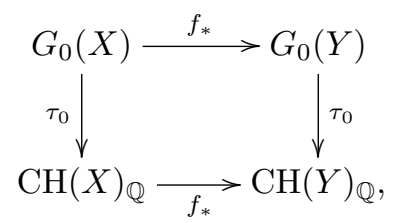

where $\tau_{0}$ is the restriction to degree zero of the natural transformation

$$
\tau: G_{0}\left[\beta, \beta^{-1}\right] \rightarrow \mathrm{CH} \otimes \mathbb{Q}\left[\beta, \beta^{-1}\right]^{(\mathrm{td})} .
$$

Moreover, $\tau_{0}$ coincides with the local Chern class morphism in [1].

Proof. The commutativity of the diagram is clear by restricting the natural transformation $\tau$ to degree zero, noting that $\tau$ is a transformation of OBM homology theories, and that the twisting construction does not alter the pushforward maps.

We claim that if $P$ is a projective space $\mathbb{P}^{n}$, the term of degree $n$ in $\tau_{0}\left(\left[\mathcal{O}_{p}\right]\right)$ is the fundamental class in $\mathrm{CH}_{n}(P),[P]$. For this, we have canonical natural transformations

$$
\Omega_{*} \stackrel{\theta_{\times}}{\longrightarrow} \Omega_{*}^{\times} \stackrel{\theta_{G}^{\times}}{\longrightarrow} G_{0}\left[\beta, \beta^{-1}\right] \stackrel{\tau}{\longrightarrow} \mathrm{CH}_{*}\left[\beta, \beta^{-1}\right]^{(\mathrm{td})} .
$$

Thus the composition

$$
\tau \circ \theta_{G}^{\times} \circ \theta_{\times}: \Omega_{*} \rightarrow \mathrm{CH}_{*}\left[\beta, \beta^{-1}\right]^{(\mathrm{td})}
$$

is the canonical natural transformation $\theta_{\mathrm{CH}}(\mathrm{td})$ given by the universality of $\Omega_{*}$. Similarly, the composition

$$
\theta_{G}^{\times} \circ \theta_{\times}: \Omega_{*} \rightarrow G_{0}\left[\beta, \beta^{-1}\right]
$$

is the canonical natural transformation $\theta_{G}: \Omega_{*} \rightarrow G_{0}\left[\beta, \beta^{-1}\right]$.

If $A_{*}$ is a OBM homology theory on $\operatorname{Sch}_{k}$, then for a cobordism cycle $[f: Y \rightarrow X]$, the canonical natural transformation $\theta_{A}: \Omega_{*} \rightarrow A_{*}$ has

$$
\theta_{A}([f: Y \rightarrow X])=f_{*}^{A}\left(1_{Y}^{A}\right) .
$$

Here $1_{Y}^{A}=p_{Y}^{*}(1)$, where $p: Y \rightarrow \operatorname{Spec}(k)$ is the structure morphism and $1 \in A_{0}(k)$ is the unit (note that by definition of a cobordism cycle, $Y$ is irreducible and in $\operatorname{Sm}_{k}$, and $f$ is projective). We use the notation $f_{*}^{A}$ to indicate the pushforward for the theory $A$.

For $A=G_{0}\left[\beta, \beta^{-1}\right]$, this gives $1_{Y}=\left[\mathcal{O}_{Y}\right] \beta^{\operatorname{dim}_{k} Y}$ and

$$
\theta_{G}([\mathrm{id}: P \rightarrow P])=\operatorname{id}_{*}\left(1_{P}\right)=\left[\mathcal{O}_{P}\right] \beta^{n} .
$$

For $A=\mathrm{CH}_{*}\left[\beta, \beta^{-1}\right]^{(\mathrm{td})}$ we have

$$
1_{Y}=\left(p_{Y}\right)_{(\mathrm{td})}^{*}\left(1_{k}\right)=\tilde{c}_{\mathrm{td}}\left(N_{p_{Y}}\right)\left(p_{Y}^{*}\left(1_{k}\right)\right)=c_{\mathrm{td}}\left(N_{p_{Y}}\right)=c_{\mathrm{td}}\left(-T_{Y}\right) ;
$$

hence

$$
\theta_{\mathrm{CH}^{(\mathrm{td})}}([\mathrm{id}: P \rightarrow P])=c_{\mathrm{td}}\left(-T_{P}\right)
$$


and thus

$$
\tau\left(\left[\mathcal{O}_{P}\right]\right)=c_{\mathrm{td}}\left(-T_{P}\right) \beta^{-n}=c_{\mathrm{td}}\left(-T_{P}\right) \quad(\text { see Remark } 4.4) .
$$

In degree 0 , this is just the classical total Todd class of $T_{P}$, which written in $\mathrm{CH}^{*}(P)$ is:

$$
\tau_{0}\left(\left[\mathcal{O}_{P}\right]\right)=\operatorname{td}\left(T_{P}\right)=\operatorname{td}\left(\mathcal{O}_{P}(1)\right)^{n+1}=\left[\frac{H}{1-e^{-H}}\right]^{n+1}=\left(1+\frac{1}{2} H+\cdots\right)^{n+1},
$$

where $H \in \mathrm{CH}^{1}(P)$ is the class of a hyperplane, and $1 \in \mathrm{CH}^{0}(P)$ is the usual fundamental class.

We conclude that $\tau_{0}$ coincides with the localized Chern class map of [1] by the following uniqueness theorem of Baum-Fulton-MacPherson.

Theorem 4.7 (Baum-Fulton-MacPherson). There is only one additive natural transformation $\phi: G_{0} \rightarrow \mathrm{CH} . \otimes \mathbb{Q}$ with the property that if $P$ is a projective space, the top dimensional cycle in $\phi\left(\mathcal{O}_{P}\right)$ is $[P]$.

Remark 4.8. The transformation $\phi$ in the above theorem being natural means it commutes with push-forwards. That is, for a projective morphism $f: X \rightarrow Y$, the following diagram:

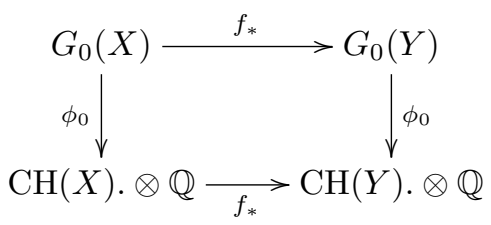

commutes.

The proof is complete.

Corollary 4.9 (Module). Let $X$ be in $\operatorname{Sch}_{k}$. Then for any $a \in K_{0}(X)$ and $b \in G_{0}(X)$, we have

$$
\tau_{0}(a \cdot b)=\tilde{\operatorname{ch}}(a)\left(\tau_{0}(b)\right)
$$

Proof. By linearity, it suffices to prove it for the case where $a=[E]$ and $b=[\mathcal{F}]$ for $E \rightarrow X$ a vector bundle and $\mathcal{F}$ a coherent sheaf on $X$. By the splitting principle, it is further reduced to the case where $E$ is a line bundle $L$, with projection $p: L \rightarrow X$. Let $\mathcal{L}$ denote the associated sheaf of sections of $L$.

We have the first Chern class operator map

$$
\tilde{c}_{1}(L): G_{0}(X)_{\beta} \rightarrow G_{0}(X)_{\beta}
$$

defined as

$$
\tilde{c}_{1}(L)(x):=s^{*} s_{*}(x) \beta^{-1},
$$

where $s: X \rightarrow L$ is the zero section. 
We resolve $\mathcal{O}_{s(X)}$, regarded as an $\mathcal{O}_{L}$-module, as follows

$$
0 \rightarrow p^{*}\left(\mathcal{L}^{\vee}\right) \rightarrow \mathcal{O}_{L} \rightarrow \mathcal{O}_{s(X)} \rightarrow 0
$$

Using the fact that the pullback map $p^{*}$ is flat we get the exact sequence

$$
0 \rightarrow p^{*}\left(\mathcal{L}^{\vee} \otimes \mathcal{F}\right) \rightarrow p^{*}(\mathcal{F}) \rightarrow s_{*}(\mathcal{F}) \rightarrow 0 .
$$

Since $s$ is a closed immersion, the higher direct images of $s_{*}$ vanish. Thus in $G_{0}(L)$, we have

$$
s_{*}([\mathcal{F}])=\left[s_{*} \mathcal{F}\right]=\left[p^{*}(\mathcal{F})\right]-\left[p^{*}\left(\mathcal{L}^{\vee} \otimes \mathcal{F}\right)\right] .
$$

Since $p$ is flat and $s^{*} p^{*}=\mathrm{id}$,

$$
s^{*}\left(\left[p^{*}(\mathcal{F})\right]\right)=[\mathcal{F}] ; s^{*}\left(\left[p^{*}\left(\mathcal{L}^{\vee} \otimes \mathcal{F}\right)\right]\right)=\left[\mathcal{L}^{\vee} \otimes \mathcal{F}\right],
$$

and we then have

$$
\tilde{c}_{1}(L)([\mathcal{F}])=s^{*} s_{*}([\mathcal{F}]) \beta^{-1}=\left([\mathcal{F}]-\left[\mathcal{L}^{\vee} \otimes \mathcal{F}\right]\right) \beta^{-1} .
$$

The naturality of the canonical transformation

$$
\tau: G_{0}(X)_{\beta} \rightarrow \mathrm{CH}_{*}(X)\left[\beta, \beta^{-1}\right]_{\mathbb{Q}}^{(\mathrm{td})},
$$

gives us

$$
\tau\left(\tilde{c}_{1}(L)([\mathcal{F}])=\tilde{c}_{1}^{(\mathrm{td})}(L)(\tau([\mathcal{F}])) .\right.
$$

Thus,

$$
\tau\left([\mathcal{F}] \beta^{-1}-\left[\mathcal{L}^{\vee}\right][\mathcal{F}] \beta^{-1}\right)=\left(\beta^{-1}-\beta^{-1} e^{-\beta \tilde{c}_{1}(L)}\right) \tau([\mathcal{F}]) .
$$

We easily deduce that, at degree 0 ,

$$
\tau_{0}\left(\left[\mathcal{L}^{\vee}\right][\mathcal{F}]\right)=e^{-\beta \tilde{c}_{1}(L)} \tau_{0}([\mathcal{F}])=\operatorname{ch}\left(L^{\vee}\right) \cap \tau_{0}([\mathcal{F}]) .
$$

One should notice that the presence of $\beta$ in $\operatorname{ch}\left(L^{\vee}\right)$ is due to the introduction of $\beta$ in the twisting of $\mathrm{CH}_{*}$-theory. Under the identification of sending $x \in \mathrm{CH}_{p}(X)$ to $x \cdot \beta^{-p}, \operatorname{ch}\left(L^{\vee}\right)$ becomes the classical Chern character of $L^{\vee}, e^{\tilde{c}_{1}\left(L^{\vee}\right)}$, which is equal to $e^{-\tilde{c}_{1}(L)}$.

The proof is then completed by replacing $L^{\vee}\left(\right.$ resp. $\left.\mathcal{L}^{\vee}\right)$ by $L$ (resp. $\mathcal{L}$ ).

\section{References}

[1] P. Baum, W. Fulton and R. MacPherson, Riemann-Roch for singular varieties, Publ. Math. l'I.H.É.S. 45 (1975), no. 1, 101-145.

[2] W. Fulton, Intersection theory, Ergeb. Math. Grenzgeb. (3) 2, Springer-Verlag, New York, 1984.

[3] W. Fulton, Rational equivalence on singular varieties, Publ. Math. l'I.H.É.S. 45 (1975), no. 1, 147-167.

[4] H. Hironaka, Resolution of singularities of an algebraic variety over a field of characteristic zero. I, II, Ann. of Math. (2) 79 (1964), no. 1, 109-203; ibid. (2) 79 (1964), no. 2, 205-326. 
[5] M. Levine, Algebraic cobordism II, preprint, June 2002. http://www.math.neu.edu/ levine/publ/AlgebraicCobordismII.pdf.

[6] M. Levine and F. Morel, Algebraic cobordism, Springer Monographs in Math., Springer-Verlag, New York, 2007.

[7] M. Levine and F. Morel, Algebraic cobordism I, preprint, Feb. 2000. http://www.math.neu.edu/ ^levine/publ/AlgCobordRev2.pdf.

[8] D. Quillen, Higher algebraic $K$-theory I, in Algebraic $K$-theory I, Lecture Notes in Math. 341 (1973), 85-147.

Shouxin Dai dai@math.ucla.edu

Mathematics Department, UCLA, 520 Portola Plaza, Los Angeles, CA 90095, USA 